\title{
Assessing Physical Internet potential for Humanitarian Supply Chains
}

\author{
Manon Grest \\ IMT Mines Albi \\ manon.grest@mines-albi.fr
}

\author{
Matthieu Lauras \\ IMT Mines Albi \\ matthieu.lauras@mines-albi.fr
}

\author{
Benoit Montreuil \\ Georgia Institute of Technology \\ benoit.montreuil@isye.gatech.edu
}

\begin{abstract}
Nowadays, Humanitarian Supply Chain stakes are changing drastically, implying a need for new methods and tools. One of the most promising evolution is definitively "Physical Internet". The current research work investigates how to assess the potential benefits and limits of using the Physical Internet paradigm within Humanitarian Supply Chains. Practically, the proposal provides (i) a systems engineering-oriented framework and (ii) a set of specific modeling features. This contribution will allow assessing efficiently and accurately, impacts of Physical Internet in Humanitarian context. Finally, the paper develops avenues for further research based on the proposal.
\end{abstract}

\section{Introduction}

When a disaster occurs a set of humanitarian actors organize to save lives and alleviate suffering. However, performance results have shown that humanitarian response lack of effectiveness to respond to the needs of affected people [1];[2]. The destabilised environment, the urgency of the situation and uncertain context are also to concede unhelpful to organize the relief operations [3]. Confronted with this observation, two options can be considered by humanitarian organizations (HOs): optimising the existing or breaking with current practices. Within the idea to act beyond current dogmas and practices, Physical Internet (PI) has recently emerged to cope with economic, social and sustainable issues in the supply chain context, seems a relevant approach for the humanitarian sector as well [4]. Indeed, by redesigning the way objects are moved, deployed, realised, supplied, designed and used, it intends to make logistics more efficient, sustainable, smart, agile, adaptable, scalable and resilient [5]. Such qualitative investigation of PI interests applied to the humanitarian sector has started to be undertaken recently by a small proportion of articles [6];[7] and so will be partially covered in this paper. However, what remains at stake in this association is to quantitatively assess the integration of PI concepts on the Humanitarian Supply Chain (HSC) performance. To conduct this, the object of interest, here HSC and called 'system', has to be studied to gain some understandings about its behavior. To this end, the investigation engages formulating assumptions and lead to a representation of the object, named 'model' [8]. Nevertheless, the modeling step is not to perform hastily if we refer to the cost of reworking when omitting a component at that stage and realizing it in the next steps [9]. Besides, few papers deal with this pairing between PI and HSC, so there is a challenge to make it properly to pave the way for further investigations and analysis. Since the HSC is a complex system including a diverse and large set of stakeholders and activities to coordinate and significantly influenced by its environment, the following question may arise: How to model a sufficiently realistic representation of HSC for future PI experiments? To answer this question, the paper aims at first, proposing a framework intended to meet this requirement level and secondly, illustrating the methodology with a first system model proposal. The article is then structured as follows. Section 2, is dedicated to give backgrounds, as an overview of the HSC context, what PI in humanitarian would mean and some insights regarding systems engineering, to help answering the previous question and support the framework establishment. Section 3 deals with the framework presentation and the context of its development. Section 4 suggests an application of the elaborated framework and provides some first modeling elements of a typical HSC. Lastly, section 5 discusses the proposals and defines avenues for further investigations.

\section{Background}

\subsection{Humanitarian Supply Chains}

Over the years, the number and complexity of disaster have not stopped increasing and such a trend 
is expected to keep going forward in future [10]. The damage caused often deprive the population of their property and resources and put their lives at risk. Needy, they can rely on HOs to alleviate their suffering as it is their mission [11]. To answer the relief demand, HOs make use of the logistics which accounts for $80 \%$ of their activities [12]. Since HOs are dealing with lives and limited time, the organization and activities completion should be the most effective as possible. Self-evident, it is much less so in practice. Indeed, the particular context and challenges whose's humanitarians have to deal with compromise the relief. To discuss this aspect, the next sections address the way HOs manage disasters and the challenges encountered during their operations.

2.1.1. Disaster Management Disaster management involves a different set of activities depending on the actual phase the emergency is [13]. Indeed, the presence of disasters introduces different timely phases forming a cycle and requiring a different type of efforts. Far before the disaster occurs, it is the mitigation phase where actions can be undertaken to strengthen population resilience. Closer to the striking, the preparedness phase involves pre-positioning means to foster the relief. As for the response phase when the disaster happens, it consists of assisting by answering basic subsistence needs. Lastly, the recovery phase, whose start-up depends on the end of the state of emergency declared by the HOs, comprises actions trying to get the population back to their initial living conditions [14]. Concretely, the preparedness and recovery phases are less undertaken by aid agencies often due to a lack of support from donors [15] while the response phase generally engages most of the HOs due to its complexity and urgency [16]. The next section details the typical humanitarian supply network as deployed during the response phase.

2.1.2. HSC Management \& Challenges When a disaster hits a territory, government and local aid agencies take immediate actions to assist the affected population. When the demand is overwhelming for locals additional help can be requested [17]. Consequently, the number of actors can quickly becoming significant and pose coordination issues [12]. Indeed, the diversity of origins of the stakeholders (i.e. situated at the local, regional, national or even outside borders [18], of roles (i.e. they can be suppliers, donors, military forces, logistics providers,... [11] and of their mission can also generate operation redundancies or lacks [19]. Besides, each event leads to the formation of a new network of actors [20] where various type of flows transit. First, to forward the relief items to the affected location, the physical flow has to transit through facilities where they may be stored for a period [21] and transported via various means. While certain are classical (e.g. airplane, trucks, sea boats,...) others are less conventional such as elephants [3] employed to reach some remote and sparsely populated areas. Numerous exchanges of information also take place in this situation to provide information and make adequate decisions. However, the high uncertainty regarding the disaster occurrence and its impacts prevent humanitarians from anticipating the demand and the means [11]. Additionally, the IT technology is not really established within the organizations while decision-makers face a lack of data reliability and accuracy [22]. Finally, regarding the financial flow, it generally comes from donors to support the organizations in their mission. However, donations usage is regularly restricted by contributors [7] reducing the scope of available resources. Also, the media play an indirect role in the complexity of relief. Indeed, the media exposure tends to multiply the perceived support and leads to unsolicited and often inappropriate donations hindering the delivery [23].

\subsection{Towards a Hyperconnected Humanitarian Supply Chain}

Given the many challenges faced by HOs in pursuing their mission and the witnessed failure in providing help after some major events [24] may question HSCs' organization. At that stage, two routes can be considered: either optimising the existing and edging toward the maturity of classical supply chains or accepting to call into question some fundamentals. It is in this second perspective that the Physical Internet may be of interests.

2.2.1. Physical Internet interests for the humanitarian sector The Physical Internet (PI) is an emergent and innovative concept of interconnected logistics networks capitalizing on the opportunity of sharing assets and capabilities [4]. It has been defined as: "[an] hyperconnected global logistics system enabling seamless open assets sharing and flow consolidation through standardized encapsulation, modularization, protocols and interfaces" [25]. As highlighted by the definition, the PI completely remodels the logistics foundations. The term 'hyperconnected' refers to the tight and intensive connection between the actors and the physical 
components of the network. This rapprochement operates through multiple layers i.e. at digital, physical operational, business, legal and personal levels thanks to an optimization of the various containers and the standardization of the exchanges [4]. The second core aspect of the PI remains in the wish of opening the logistics networks and sharing the assets. Nowadays, companies are part of a specific and stable network and possess their warehouses or fleets. The concept takes the opposite of this view and induces that assets should be shared between all the users part of this global network and used on a need basis. Also, the PI as 'the network of networks' would increase the visibility and opportunity for new partnerships. Such a concept of PI appears as a result of the awareness that only a new logistics organisation will enable meeting the environmental and service requirement challenges. Indeed, the current logistics system shows too many unsustainable symptoms and is participating in compromising the Paris agreements objectives [5]. Before it is too late, the PI has been designed to offer a more resilient, efficient, sustainable and adaptable logistics service to its users by changing the way physical objects are handled along the network [4]. As an illustration, sharing assets would optimize the utilization rate and allows cost-savings and reducing $\mathrm{CO} 2$ emissions as well as interconnecting actors would certainly allow speeding up the physical, financial and information flows along with the network [26].

\subsubsection{Hyperconnected humanitarian supply chain}

This part aims at highlighting the perspective of a PI approach applied to humanitarian logistics to reach a more effective, efficient and sustainable operating model. Considering the challenges faced by humanitarian, there are several fields where the PI approach could be valuable. First, as in classical supply chains, the opportunity to share means would optimize the storage and transport capacity and authorize some cost-savings. Besides, mutualizing stocks from different organizations inside the same warehouse would also increase the coverage of each actor and so, its responsiveness to deploy items and reducing delivering lead times [6]. Consolidating transports (i.e. fulfilling trucks with deliveries from different HOs) would increase the utilization rate while decreasing the number of trucks sent and so, reducing the costs and the $\mathrm{CO} 2$ emissions [6]. Another key domain where the PI may intervene is in the connection of actors. Indeed, by interconnecting the various humanitarian stakeholders the communication would be certainly improved. Besides, the development of a centralized digital platform, as suggested by Abdoulkadre et al., would solve the operations inefficiency and redundancies issues. Indeed, by centralizing and sharing in real-time information to all the key players regarding the situation would probably improve the accomplishment of the decisions made [7]. Performance would be monitored from a global relief point of view and not from an organizational one anymore. Consequently, the coordination and collaboration between actor are expected to be improved as well. Such a platform could also help in donation management. Indeed, by centralizing the need appeals, donations would be registered as humanitarian assets and based on the utilization tag given by the contributors would be deployed to the closest needed locations [7], leading to an increase in resource availability. Lastly in this overview, the concept of an open global network would also provide HOs with more available assets and resources by taking opportunities from the private sector means. Consequently, cost-savings, CO2 emission and delivery lead time reduction and even increased availabilities and responsiveness can be, among other things, expected. As mentioned previously, this is just an overview because the topic of a hyperconnected humanitarian supply chain is recent and gathers only a small portion of papers mentioned in this section. There is still a lot of investigations to lead to define what a hyperconnected humanitarian supply chain would mean but the perspectives seem attractive for the sector.

\subsection{System Engineering}

Nowadays, the number of complex systems is increasing. Indeed, they have to interact with a rich variety of other systems, serve a variety of purposes and meet increasing performance requirements such as reliability or stability etc. Consequently, such systems are more complicated to develop and produce since they require the coordination of a large number of contributors from diverse disciplines [27]. Besides, reports have shown, but a similar idea can be applied to most type of project, that only $29 \%$ of software development projects were successful (i.e. the system met the requirements) [28]. Based on these observations, it appears that attention must be paid on the need definition and the system design as they can be the source of some cost and time savings [9].

To help in that sense, the field of system engineering (SE) has emerged around 1970 and is increasingly attracting the attention of both researchers [27] and engineers [29]. Intended to deal with complex and interdisciplinary systems and enabling their successful realization [27], SE is based on a holistic approach 
(i.e. considers the system as a whole and the interactions with its environment). It also captures the whole identity of the system via a description from a different point of views [27]. Besides, it presents the advantages of enhancing the communication between the stakeholders by providing a shared understanding of the system thanks to a collection of standardized modeling languages and also structuring the system conception with a process-based approach [29]. Indeed, each step of the system life cycle (i.e. from the business requirements to the system deployment) is described as a process by the ISO/IEC/IEE15288:2015 [30]. Finally, SE presents the aspect of integrating validation and verification actions through all steps of the life cycle. Respectively defined as, "the set of activities ensuring and gaining confidence that a system is able to accomplish its intended use, goals and objectives (i.e., meet stakeholder requirements) in the intended operational environment. The right system was built." and "the set of activities that compares a system or system element against the requirements, architecture and design characteristics, and other properties to be verified" [30], those regular interventions guarantee the compliance of the system with its purpose.

\section{Proposal}

\subsection{Generic framework}

In the perspective to assess the impact of PI approach applied to the HSC field and draw reliable and objective conclusions, and even before undertaking any experiments, we have to ensure the validity and credibility of the model developed.

On one hand, according to Law, credibility comes from the approval of the client about the correctness of the system behavior regarding reality. However, being credible does not guarantee that the model is valid while validating helps to establish credibility [8].

On the other hand, the subject to model, here HSC, is admitted to be complex in the light of Sheard and Mostashari's categories developed as follows [31]. It is first complex by its structure. As a matter of fact, a multitude of various actors are part of the HSC and induces the existence of many type of assets (e.g. warehouses, fleet...) and communications to supply people in need. Secondly, complexity comes from the dynamic of the system. Many logistics activities are performed and adjust according to the environment influence (e.g. a destroyed road leads to rerouting...) and the phases. Finally, the presence of media, donors and government influencing the relief brings additional socio-political complexity to the system.
Consequently, based on (i) the HSC complexity generated from external (i.e. environment, socio-politics,...) and internal (e.g. humanitarian actors, assets,...) factors as well as all the flows resulted in and (ii) the research needs for validity and those, put into perspective with SE properties allowing dealing with complex, multi-disciplinary system while ensuring the verification \& validation of the system throughout its life cycle phases, highlight the SE approach as a good solution for properly modeling the HSC system. Thereupon, we developed a framework based on the SE System Definition process (See Figure 1). It also preserves the property of SE that consists of having validity actions at every step. It encompasses three stages namely, the system mission, the system architecture and the system design. The first step aims at defining the problem addressed by the system and its context. It is intended here to end up with the need(s) description and validation. The system architecture paints a picture and validate (i) the purpose of the system and its context, (ii) the logical architecture (i.e. its behavior scenario) and (iii) the physical architecture where the system elements are explained. The last step gives indications for the element execution for the next phases such as the implementation that is not covered in this article.

\subsection{Humanitarian Supply Chain features}

In this section, to get a better insight into the framework suggested, we propose to put it into action and deliver some first modeling elements of a typical HSC.

3.2.1. Step 1 - System Mission Starting with defining the problem addressed by the system and its context, the global study focuses on the assessment of a PI approach on HSC performance. Before being able to experiment some PI change by playing with variables or parameters and assessing the impacts, it appears logical to gain some understandings first about the current system and builds up a model. Given its complexity, to represent its behavior the formulation of a set of assumptions is required [8]. Besides, to draw objective conclusions at the end of the study, we have to ensure the reliability of the representation and this is often one of the biggest challenges [32].

\subsubsection{Step 2 - System Architecture}

\section{- System Context Definition}

Before going further in the details, the global definition 

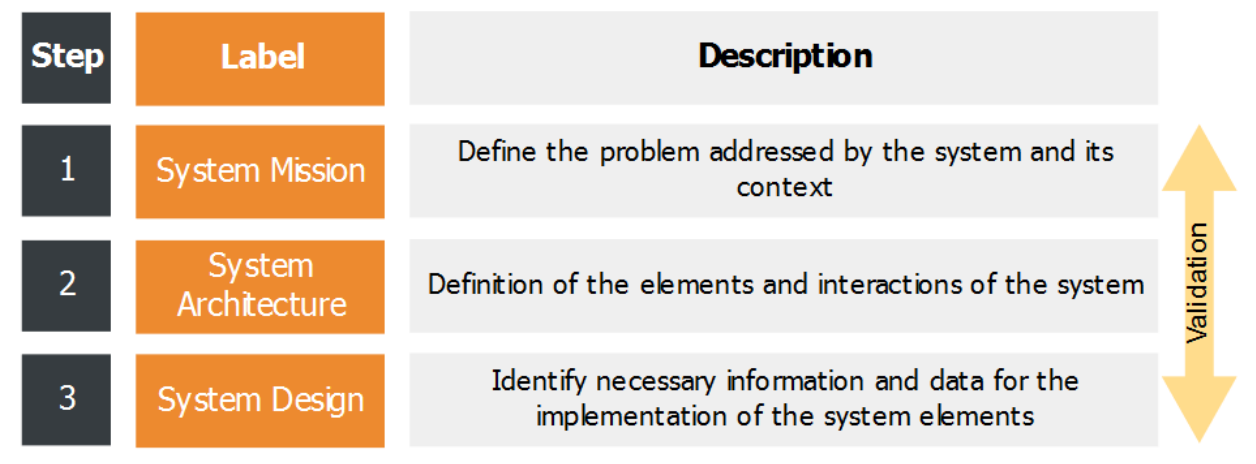

Figure 1. Framework suggestion for modeling HSCs

of a system is required. According to the International Organization for Standardization (ISO), a system is "a combination of interacting elements organized to achieve one or more stated purposes" [30]. An 'element' is defined as a component of a system that may be also part of a subsystem. The term 'interaction' refers to the manner element influence each other. Finally, the 'purpose' appears to meet a need and can be declined into three objective typologies such as the performance (e.g. reliability, capacity, usability,...), the costs (e.g. price, ROI,...) or the lead time (e.g. durability, milestones,...) [30] To start defining the system, we can rely on one of the most cited definitions related to HSC. Indeed, since logistics accounts for $80 \%$ or relief operations [12], it provides a first focus angle to the model. Then, according to Kovacs et Spens 2012, it "encompasses the planning and management of all activities related to material, information and financial flows in disaster relief. Importantly, it also includes coordination and collaboration with supply chain members, third-party service providers, and across humanitarian organizations [13]. Based on this, it stands out that: (i) elements are the logistics activities and the various actors (ii) interactions are represented through the three types of flows and the notion of coordination and collaboration and (iii) the purpose stands in the 'disaster relief' that encompasses the main mission of humanitarian known as, saving lives and alleviating suffering [1]. At that stage, the 'internal' description of the system of interest, that will be named the 'humanitarian response system', is made.

Then, as suggested by the holistic approach, the description of the environment and its influence on the system needs to be covered. To that end, if we rely on Kovacs and Spens (2009)'s article related to the identification of humanitarian logistics challenges and offering a conceptual model for program development, it appears that: "the actual challenges faced in a disaster depend on the type of the disaster as well as the region the disaster occurs in" [33]. They also identify the different phases of the disaster relief have an impact on the logistics activities [33] in other words, the dynamic after the disaster. Lastly, the two other challenges detected (i.e. the variety of humanitarian organizations and the coordination of actors) strengthen the need for the presence of those two components in the model. From this first investigation on the key components of HSC, we developed an illustration providing an overview of the HSC system context (See Figure 2). The HSC system context contains the 'humanitarian response' system which is the system of interest (SoI) [29] interacting with the demand system part of the wider system and including the SoI as well. Then, arranged around the latter, the 'territory' and 'disaster' systems (i.e. the external systems part of the environment influencing the wider System [29].

\section{- Logical Architecture}

In this part, the objective of the logical architecture is to describe the behavior of the system as it operates in reality [29] (See Figure 3). Basically, according to Thomas and Kopczak (2005), once a disaster occurs at a location, it makes damage and victims. The people affected need relief and generate a demand for items to supply. For this reason, a system to deal with the demand, called the 'demand system' is required. From that moment, starts the response phase, aid agencies are active through their logistics activities and thus, mobilize stock, organize transportation and deliveries [24]. The influence of the supply on the demand system needed to appear as HSCs performance is a decisive factor to save lives [3]. Such a typical scenario only applies from the response to the recovery phases. As for the preparedness stage, it relates to the anticipation of needs by pre-positioning means at strategic locations but it is not always performed by humanitarian organizations due to the difficulties to assess and anticipate the future impacts [3] 


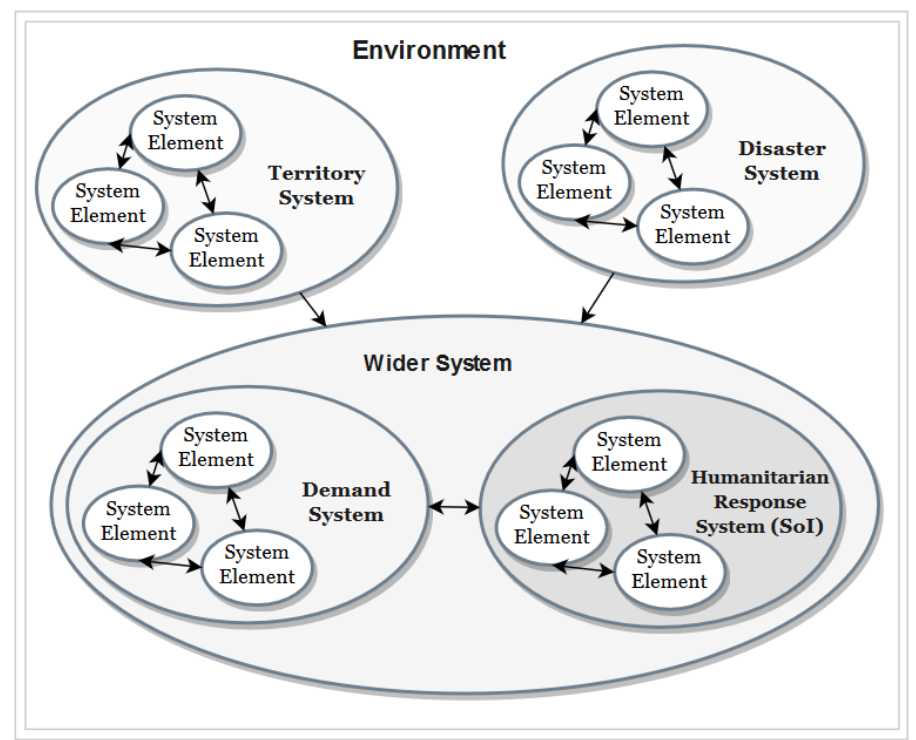

Figure 2. HSC Features: the System Context - Adapted from the general description of System Context (SEBoK original) illustration

\section{- Physical Architecture}

In this second part related to system physical architecture, the objective is to depict each subsystem in terms of objective and elements [29].

\section{- Territory System}

The territory system is part of the environment of the SoI. It evolves continuously over time and represents a territory susceptible to be affected by a disaster. It can be a country or smaller piece in the administrative breakdown like a province or an island. That is why it may be necessary to specify the administrative breakdown structure. The territory can be represented as an overlay of layers (See Figure 4) namely, the topography, the population, the infrastructures and the transport network. The topography may be of some influence in the delivery speed or road state and lead to unconventional delivery systems that is why it has been integrated [23]. The population is obviously required as it determines the demand of items as well as the buildings such as houses or public structures that induces the need for specific supplies like tents or medicines. At last, the transport network integrates two sub-elements: (i) the transport roads encompassing the different type of roads (car, sea, air,... road) and (ii) the transport nodes connecting the roads with seaports, airports etc. All of them are followed by transportation and delivery activities.

- Disaster System
The disaster system constitutes the second side of the environment of the SoI. It is a discontinuous system representing events hitting the territory and provoking damages. To reproduce the impact notion, key characteristics of a disaster (e.g. type, intensity,...) and its dynamic during the entire period of its existence are required.

\section{- Demand System}

The demand system is a related system of the SoI. It activates when a zone requires humanitarian assistance and generates a demand for items or service to aid agencies for the whole duration of the emergency state. It consists of two interacting subsystems: the 'human and material impact system' and the 'requirement system'. The first one aims at providing figures regarding the number of victims and damages over time. The second indicates the specific needs associated with an event. Both of them require inputs from the environment to operate and together they assess the relief demand. Such system encompasses the work performed by assessment teams. It is a choice to have it separated from the humanitarian response system since it is not always considered as a relief logistics activity but more as an input. Besides, it has also been set apart to introduce the influence notion of the humanitarian response on it. Indeed, it is admitted that the speed of the response or a delay in the supplies influence the number of lives saved [3].

- Humanitarian Response System 


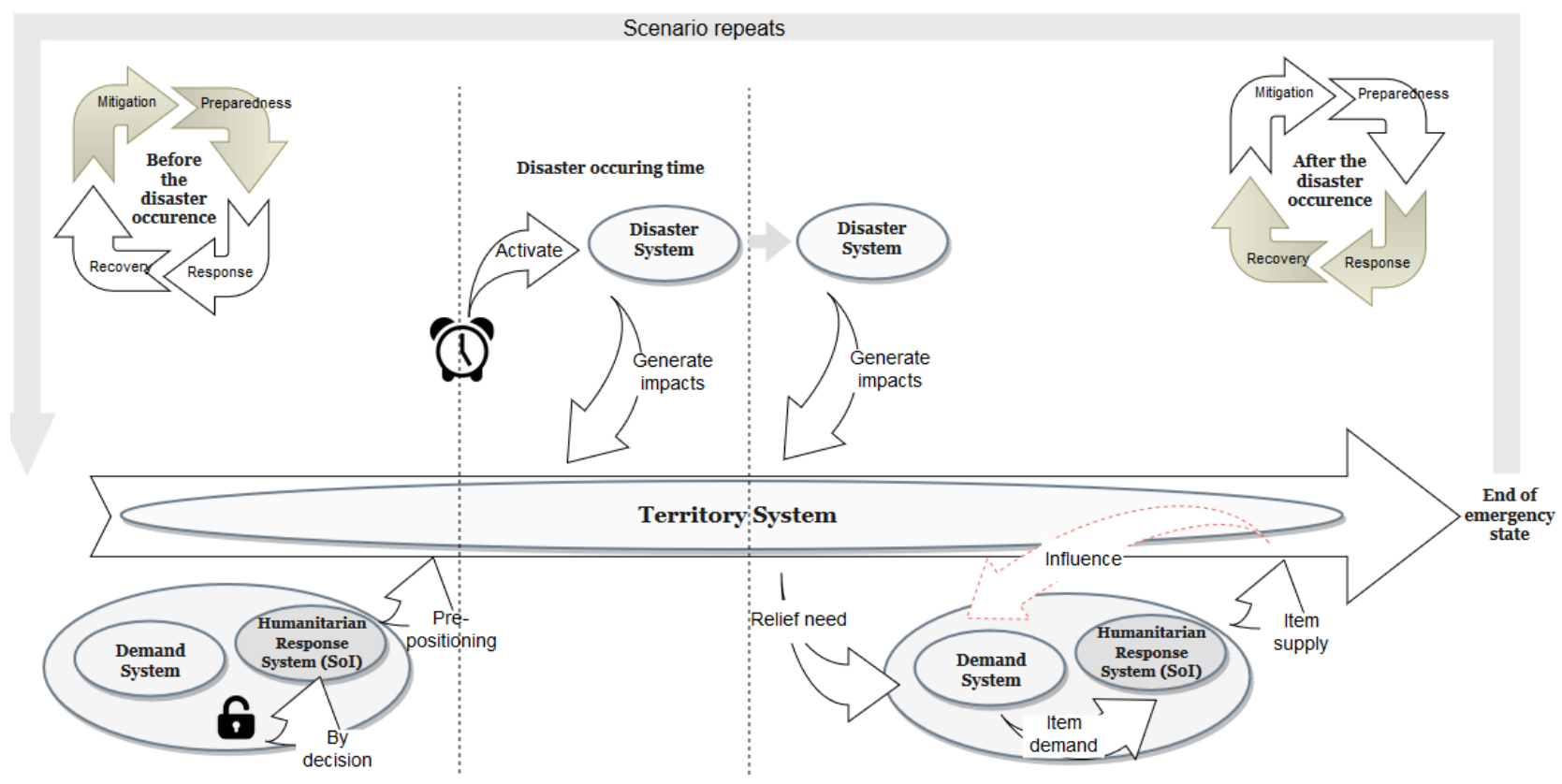

Figure 3. HSC features: the Logical Architecture

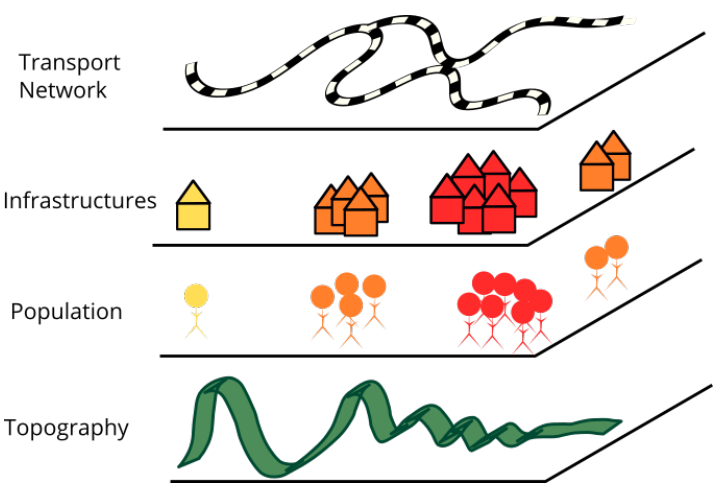

Figure 4. HSC Features: the Territory modeling with layers

The humanitarian response system aims at representing HSCs establishment after a disaster appearance. As seen previously in the System Context definition, the key elements of such a system are the actors (e.g. aid agency, government, donors, suppliers,...), the containers to encapsulate the products from the cardboard boxes to the transportation means (e.g. trucks, boats,...) and the storage assets. The interactions between those components are due to the physical, information or financial flows driven by the different logistics activities.

3.2.3. Step 3 - System Design Normally, the objective of the System Design is to provide useful indications for the implementation of the system elements [29]. In that sens and before that, we will only highlight the data collection and achievements to perform. Regarding the data to gather to get realistic modeling, information regarding the administrative division breakdown, topology, transport nodes and roads characteristics, population features and disaster history of a given territory are expected. Then, information needs to be gathered regarding the supplier's profile, humanitarian actors and storage assets distributed over the territory as well. As achievements, we mean investigations to lead in the perspective to gain additional understandings in some behaviors. Indeed, some issues are currently faced due to a lack of explanations in the available literature. For instance, in the case of the demand system, current disaster databases are only providing the total number of victims and losses after each event. No information has been found regarding the evolution of damages overtime after a natural disaster and so, missing for generating a dynamic demand and fueling the humanitarian response system as it is the case in reality. Also, there is a lack of accuracy in the way aid agencies are responding to disaster (e.g. how they make decisions or prioritization, how they sequence activities,...) and that complicates the modeling the response process.

\section{Conclusion and perspectives}

In research, making experiments generally involves modeling the system of interest beforehand. However, 
as well as marketed systems gain in complexity, the same reality happens in the systems to investigate and the HSC is not an exception. Besides, the scientific approach requires to ensure the validity of the model as a reliable representation of the system despite the assumptions made. Considering these two constraints and knowing the importance of the modeling phase for the aftermath of the study, some reflections regarding the way to proceed had to be examined.

The System Engineering domain developed to deal with complex systems, integrating multi-disciplinary components while ensuring the realization of success and the adequacy of the system came out as a suitable approach. Taking inspiration from it, we developed a framework to structure the modeling of the HSC and satisfy the requirements previously outlined. Then, some first representative elements of a typical HSC are also provided as a result of the application of the defined guidelines.

However, one step of the SE process is missing in the approach issued, namely the 'System Analysis'. This phase aims at quantitatively assessing the system to end up with the most appropriate system architecture [29]. This phase has not been covered yet but highlights the fact that the model is subject to some enrichment as the research progress. Indeed, according to the classical research process, the next step would be to design the experiment. Defining the variables influencing the outcomes of the study may lead to the addition of some missing components to the model. For instance, in a PI sense, we may want to forecast the demand before the disaster occurs to improve the coverage. Such an idea would probably require to investigate the vulnerability of territories and so, requiring some updates.

Finally, as identified by Behl and Dutta as a literature gap in the field of humanitarian supply chain management, additional investigations are required to standardize models and also capture the dynamic to analyse cause and effect relationship [34].

\section{References}

[1] I. Haavisto and J. Goentzel, "Measuring humanitarian supply chain performance in a multi-goal context," Journal of Humanitarian Logistics and Supply Chain Management, vol. 5, pp. 300-324, Dec. 2015.

[2] C. J. Chiappetta Jabbour, V. A. Sobreiro, A. B. Lopes de Sousa Jabbour, L. M. de Souza Campos, E. B. Mariano, and D. W. S. Renwick, "An analysis of the literature on humanitarian logistics and supply chain management: paving the way for future studies," Annals of Operations Research, vol. 283, pp. 289-307, June 2017.

[3] R. Tomasini and L. V. Wassenhove, Humanitarian Logistics. Springer, Feb. 2009. Google-Books-ID: E72GDAAAQBAJ.

[4] B. Montreuil, R. D. Meller, and E. Ballot, "Physical
Internet Foundations," IFAC Proceedings Volumes, vol. 45, pp. 26-30, May 2012.

[5] E. Ballot, L'Internet physique: le reseau des reseaux des prestations logistiques. Transports, recherche, innovation, Paris: la Documentation franaise, 2014.

[6] M. Grest, M. Lauras, and B. Montreuil, "Toward Humanitarian Supply Chains Enhancement by using Physical Internet Principles," 8th International Conference on Industrial Engineering and Systems Management, Sept. 2019.

[7] A. Abdoulkadre, B. O. Intissar, M. Marian, and M. Benoit, "Towards Physical Internet Enabled Interconnected Humanitarian Logistics," 1st International Physical Internet Conference, p. 30, May 2014.

[8] A. M. Law, Simulation Modeling and Analysis. Dubuque: McGraw-Hill Higher Education, 5 ed., Feb. 2014.

[9] B. Boehm, "Software Engineering," IEEE Transactions on Computers, vol. C-25, pp. 1226-1241, Dec. 1976. Conference Name: IEEE Transactions on Computers.

[10] A. Cozzolino, Humanitarian Logistics: Cross-Sector Cooperation in Disaster Relief Management. SpringerBriefs in Business, Berlin Heidelberg: Springer-Verlag, 2012.

[11] G. Kovacs and K. M. Spens, "Humanitarian logistics in disaster relief operations," International Journal of Physical Distribution \& Logistics Management, vol. 37, pp. 99-114, Mar. 2007.

[12] L. N. Van Wassenhove, "Humanitarian aid logistics: supply chain management in high gear," Journal of the Operational Research Society, vol. 57, pp. 475-489, May 2006.

[13] G. Kovacs and K. M. Spens, eds., Relief supply chain management for disasters: humanitarian aid and emergency logistics. Hershey, PA: Information Science Reference, 2012.

[14] R. D. Kusumastuti, R. Insanita, and S. S. Wibowo, "Relief Logistics Practices in Indonesia: A Survey," SSRN Scholarly Paper ID 1681217, Social Science Research Network, Rochester, NY, Sept. 2010.

[15] I.-W. Kwon and S.-H. Kim, "Humanitarian Supply, Chain/Logistics: Roadmap to Effective Relief Effort," Journal of International \& Interdisciplinary Business Research, vol. 5, p. 17, June 2018.

[16] D. P. Coppola, Introduction to International Disaster Management. Elsevier, 3rd ed., 2015. Google-Books-ID: s6oxEraqWWwC.

[17] T. G. Weiss, Humanitarian Intervention. John Wiley \& Sons, May 2016. Google-Books-ID: grsvDAAAQBAJ.

[18] N. Altay, G. Heaslip, G. Kovcs, K. Spens, P. Tatham, and A. Vaillancourt, "Innovation in Humanitarian Supply Chains: A Systematic Review," Cirano, p. 27, Mar. 2018.

[19] J. Seaman, "Malnutrition in Emergencies: How Can We Do Better and Where Do the Responsibilities Lie?," Disasters, vol. 23, no. 4, pp. 306-315, 1999.

[20] V. Salaun, "Les logistiques temporaires : faire vite en prenant son temps," Supply Chain Magazine, pp. 80-81, May 2017.

[21] M. S. Pishvaee, F. Jolai, and J. Razmi, "A stochastic optimization model for integrated forward/reverse logistics network design," Journal of Manufacturing Systems, vol. 28, pp. 107-114, Dec. 2009. 
[22] B. M. Beamon and B. Balcik, "Performance measurement in humanitarian relief chains," International Journal of Public Sector Management, vol. 21, pp. 4-25, Jan. 2008.

[23] A. Charles, M. Lauras, and L. Van Wassenhove, "A Model to Define and Assess the Agility of Supply Chains: Building on Humanitarian Experience," International Journal of Physical Distribution \& Logistics Management, vol. 40, pp. 722-741, Sept. 2010.

[24] A. Thomas and L. Kopczak, "From logistics to supply chain management: the path forward in the humanitarian sector," 2005.

[25] B. Montreuil, "Sustainability and Competitiveness Is the Physical Internet a Solution?," July 2017.

[26] B. Montreuil, "Toward a Physical Internet: meeting the global logistics sustainability grand challenge," Logistics Research, vol. 3, pp. 71-87, May 2011.

[27] A. L. Ramos, J. V. Ferreira, and J. Barcelo, "Model-Based Systems Engineering: An Emerging Approach for Modern Systems," IEEE Transactions on Systems, Man, and Cybernetics, Part C (Applications and Reviews), vol. 42, pp. 101-111, Jan. 2012.

[28] S. Group, “CHAOS Report,” tech. rep., Standish Group International, Inc, 2015.

[29] R. Cloutier, ed., The Guide to the Systems Engineering Body of Knowledge (SEBoK). Hoboken, NJ: SEBoK Editorial Board, v. 2.2 ed., May 2020.

[30] ISO, IEC, and IEEE, ISO/IEC/IEEE 15288:2015 Ingenierie des systemes et du logiciel - Processus du cycle de vie du systeme. 2015.

[31] S. A. Sheard and A. Mostashari, "Complexity Types: From Science to Systems Engineering," INCOSE International Symposium, vol. 21, no. 1, pp. 673-682, 2011.

[32] B. Montreuil, C. Cloutier, O. Labarthe, and J. Loubier, "Holistic modelling, simulation and visualisation of demand and supply chains," International Journal of Business Performance and Supply Chain Modelling, vol. 7, pp. 53-70, Jan. 2015. Publisher: Inderscience Publishers.

[33] G. Kovacs and K. Spens, "Identifying challenges in humanitarian logistics," International Journal of Physical Distribution \& Logistics Management, vol. 39, pp. 506-528, Jan. 2009. Publisher: Emerald Group Publishing Limited.

[34] A. Behl and P. Dutta, "Humanitarian supply chain management: a thematic literature review and future directions of research," Annals of Operations Research, Mar. 2018. 